Recepción: 13 / 02 / 2018

Aceptación: 09 / 04 / 2018

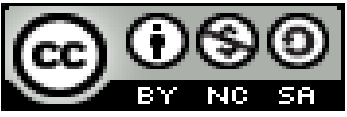

Ciencias de la salud

Publicación: 01 / 08 / 2018

Artículo de investigación

\title{
Tratamiento del duelo en pacientes diabéticos con diagnóstico de amputación
}

\section{Grief Treatment in diabetic patients with amputation diagnosis}

\section{Tratamento do luto em pacientes diabéticos com diagnóstico de amputação}

Delia G. Bravo-Bonoso ${ }^{\mathrm{I}}$

deliabravo85@hotmail.com

Dóris S. Delgado-Bernal ${ }^{\mathrm{II}}$

deigado.susana@hotmail.com

Eddy Rigoberto-Mendoza ${ }^{\text {III }}$ rodriguez..rigo747@ hotmail.com

Correspondencia: deliabravo85@hotmail.com

\footnotetext{
${ }^{\text {I }}$ Magister en Emergencias Médicas, Licenciada en Ciencias de la Enfermería, Docente de Universidad Estatal del Sur de Manabí, Jipijapa, Ecuador.
${ }^{\text {II }}$ Magister en Gerencia de Salud para el Desarrollo Local, Licenciada en Enfermería, Docente Universidad Estatal del Sur de Manabí, Jipijapa, Ecuador.

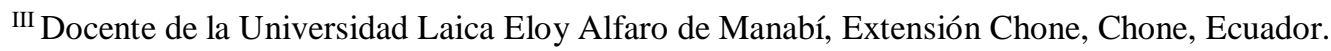




\section{Resumen}

Esta investigación y sus resultados son de carácter innovador por su aporte significativo a la población afectada, en el desarrollo de la misma se contó con el acceso a la información de manera directa con los sujetos de estudio, garantizando confiabilidad en la información proporcionada por los pacientes, actualmente la tendencia al incremento de las enfermedades crónicas no transmisibles, dan cuenta de un contexto epidemiológico donde se espera que exista un aumento sostenido de las enfermedades que constituyen las principales causas de amputación como la diabetes, en adultos mayores de 40 años. El objetivo es identificar el proceso del duelo que sufren las personas que afrontan alguna amputación de una extremidad. La investigación es cualitativa y busca una comprensión particular del fenómeno que se estudia, entiende el significado de aquello que se muestra en lo que es diferente de los hechos, se centra en lo específico, individual y peculiar. La comprensión del fenómeno es más próxima a la vivencia del paciente y la percepción de su autovalencia futura, tomando en cuenta que el resultado permitió un acercamiento al grupo poblacional con necesidades de atención, mediante la investigación se pudo observar diferentes sentimientos entre ellos vergüenza y enojo mayormente, externalizados.

Palabras clave: envejecimiento; amputación; diabetes; duelo.

\section{Abstract}

This research and its results are of an innovative nature due to their significant contribution to the affected population, in the development of which they had access to information directly with the study subjects, guaranteeing reliability in the information provided by the patients. , currently the tendency to increase chronic no communicable diseases, account for an epidemiological context where it is expected that there is a sustained increase in diseases that are the main causes of amputation such as diabetes, in adults over 40 years. The objective is to identify the process of mourning suffered by people who face some amputation of a limb. The research is qualitative and seeks a particular understanding of the phenomenon being studied, understands the meaning of what is shown in what is different from the facts, focuses on the specific, individual and peculiar. The understanding of the phenomenon is closer to the patient's experience and the perception of their future self-valence, taking into account that the result allowed an approach to the population 
group with attention needs. Through the investigation it was possible to observe different feelings among them shame and Anger mostly, outsourced.

Keywords: aging; amputation; diabetes; mourning.

\section{Resumo}

Esta pesquisa e seus resultados são inovadores por sua contribuição significativa para a população afetada no desenvolvimento de que ele teve acesso a informações diretamente com os sujeitos do estudo, assegurando a confiabilidade das informações fornecidas pelos pacientes a tendência para um aumento das doenças não transmissíveis crónicas representam actualmente um contexto epidemiológicas em que é esperado um aumento sustentado em doenças que são as principais causas de amputação como diabetes em adultos com mais de 40 anos. O objetivo é identificar o processo de luto sofrido por pessoas que enfrentam alguma amputação de um membro. A pesquisa é qualitativa e busca uma compreensão particular do fenômeno a ser estudado, ele entendeu o significado do que é mostrado no que é diferente dos fatos, com foco no específico, individual e única. Compreender o fenômeno está mais perto de experiência e percepção de seu futuro autovalencia do cliente, tendo em conta o resultado permitiu uma abordagem aos cuidados de grupo as necessidades da população através de pesquisa pudessem observar sentimentos diferentes, incluindo vergonha e raiva principalmente, terceirizado.

Palavras chave: envelhecimento; amputação diabetes; duelo.

\section{Introducción}

La discapacidad por amputación se incrementa por enfermedades como la diabetes mellitus (DM), especialmente de tipo 2, cuyo aumento es alarmante en todas las latitudes. Se estima que las personas que presentan esta condición, tienen más probabilidad de sufrir este procedimiento que los no diabéticos (Organización Mundial de la Salud, 2011), (OMS). Además, existen otras causas de amputación como en el cáncer de hueso u osteosarcoma, en la enfermedad vascular referida a la trombosis venosa profunda, sobre todo a nivel de extremidades inferiores, así como los producidos a consecuencia de accidentes de tránsito e industriales, que actualmente crece significantemente en nuestro medio (Díaz, 2015). 
Según datos recopilados por la OMS (2004), en el mundo 450 millones de personas presentan problemas en su salud mental, 347 millones de personas viven con diabetes, mientras que el $40 \%$ de la población mundial mayor de 25 años padece algún grado de hipertensión arterial.

Es relevante destacar lo señalado por la Organización Mundial de la Salud, que la proporción de personas con discapacidad oscila entre el $10 \%$ y $12 \%$ del total de la población mundial; encontrándose en éste universo las personas que han sufrido la pérdida de una o más extremidades a causa de la Diabetes Mellitus tipo II, la cual es un conjunto de trastornos metabólicos, cuya característica común principal es la presencia de concentraciones elevadas de glucosa en la sangre de manera persistente o crónica, debido ya sea a un defecto en la producción de insulina.

Es eminente la frecuencia de depresión en pacientes diabéticos. Provocando, en estas personas, diversos cambios en su estilo de vida que son frecuentemente estresantes. Con el paso del tiempo, estas tensiones y sentimientos negativos pueden robarle la energía emocional necesaria para las actividades diarias. La falta de progreso en su recuperación o el empeoramiento de los síntomas pueden desencadenar pensamientos que aumentan los sentimientos de ansiedad, tristeza y culpa que llevan a la depresión, en pacientes con complicaciones diabéticas.

La principal causa de mortalidad en todo el mundo, según las estimaciones de la Organización Mundial de la Salud (OMS) (2016), 422 millones de adultos en todo el mundo tenían diabetes en el 2014, frente a los 108 millones de 1980. La prevalencia mundial (normalizada por edades) de la diabetes casi se ha duplicado desde ese año, pues ha pasado del 4,7\% al 8,5\% en la población adulta. En el 2013 se registraron 63.104 defunciones generales, la principal causa de muerte es la diabetes mellitus, con 4.695, según información del Instituto Nacional de Estadística y Censos (INEC), (2014). (Díaz, 2015) y (Sani, 2017).

El estilo de vida desempeña un papel fundamental en la etiología de la diabetes y es el motivo por el que la diabetes tipo 2 contribuye al menos entre el 90 y el $95 \%$ al aumento de la diabetes en la población mundial. La adopción del estilo de vida occidental en los países en desarrollo ha aumentado y ha cambiado el patrón de vida cotidiano de muchos países. La falta de tiempo, que viene determinada por la creciente presión en el ámbito laboral, ha implicado que se reduzca 
notablemente el tiempo que se debe dedicar a la preparación de comidas sanas que se eleva el riesgo de contraer una diabetes de tipo 2.

Las personas que padecen diabetes deben lidiar con algunos de los problemas que trae aparejada esa enfermedad, en muchas ocasiones se ha observado que, por falta de conocimientos, no han podido acceder a los servicios y sobre todo una información del problema y los peligros que esta enfermedad conlleva, la vivencia diaria de los pacientes que son sometidos a amputaciones, los lleva a identificarse como una persona incapaz de poder desenvolverse en el medio y a su vez lo ven como una condición de vida no aceptada en la sociedad, además tiene un profundo impacto físico y emocional a lo largo del proceso crónico degenerativo propio de la enfermedad que afecta la calidad de vida, en su satisfacción con su situación física, vida familiar, social, así como el sentido que le atribuye a su vida, nadie más para comprender el dolor interno y externo que se siente por la pérdida de un miembro al cual se ha adaptado toda su vida, si no es el mismo paciente que debe pasar por este duro y largo proceso de transición de la enfermedad, los mismo que al no contar con los suficientes recursos se sienten imposibilitados de realizar acciones directas para mitigar en parte factores de riesgo, de aquí se parte con una información hacia los cuidados paliativos en los pacientes diabéticos.

Trabajar las emociones y el dolor de la pérdida de un miembro, el dolor físico literal que mucha gente experimenta y el dolor emocional y conductual asociado con la pérdida es significativo en el proceso. Puede haber una sutil interacción entre la sociedad y la persona, cada individuo muestra su propio esquema personal referido a las reacciones ante sus pérdidas. La negación funciona como un amortiguador después de una noticia inesperada o impresionante, permite recobrarse al paciente y, con el tiempo, movilizar otras defensas menos radicales, tomando en cuenta que generalmente la negación es una defensa provisional y pronto podrá ser sustituida por una aceptación.

La prevalencia mundial (normalizada por edades) de la diabetes casi se ha duplicado desde ese año, pues ha pasado del 4,7\% al 8,5\% en la población adulta. En Ecuador, en el año 2015 el $7.05 \%$ de las defunciones fueron por diabetes (Sani, 2017). Ecuador es uno de los países de Latinoamérica con el más alto índice de la enfermedad, especialmente la provincia de Manabí, en la Parroquia Picoazá de la zona urbana de Portoviejo, registra más casos de diabetes tipo II, 
llegando al $12 \%$ de lo establecido, es decir, sobrepasa el $8 \%$ que se considera; la diabetes está afectando a la población con tasas cada vez más elevadas, evidenciando que la prevalencia es la obesidad, la que cada vez aumenta en todos los grupos de edad. 3 de cada 10 niños en edad escolar presentan sobrepeso y obesidad. 1 de cada 4 niños en edad preescolar es pequeño para su edad y el porcentaje del sobrepeso se ha duplicado en las últimas tres décadas. 2 de cada 3 ecuatorianos entre los 19 y 59 años tiene sobrepeso y obesidad, lo que constituye un serio problema de salud pública.

El enfoque es comparar las estrategias de afrontamiento, aceptación y/o negación del duelo en pacientes que hayan tenido pérdidas originados por una causa médica, definiendo un marco teórico-conceptual, que identifique los posibles factores que influyen, directa o indirectamente, en la forma como se lleva a cabo el proceso de duelo en pacientes diabéticos amputados como consecuencia de situaciones médica específica y proponer alternativas de intervención terapéutica intrahospitalarias y extra hospitalarias que permitan el abordaje integral temprano para la elaboración de proceso de duelo en especial en pacientes que hayan sido sometidos a una amputación quirúrgica.

La amputación es un procedimiento quirúrgico, que se realiza por causas que pueden ser congénitas, traumáticas o debido a enfermedades, tales como la enfermedad vascular, diabetes mellitus, cáncer e infecciones, donde la única solución es retirar el miembro que ocasiona dolor, respecto a la clasificación de las amputaciones, pueden agruparse en amputaciones menores, en las que solo se amputa un dedo del pie; y las amputaciones mayores, en que se amputa parte de la pierna.

Destacar que en toda amputación el objetivo es eliminar todo el tejido infectado e isquémico e intentar preservar la funcionalidad de la extremidad al máximo. A pesar de que para una recuperación y rehabilitación óptimas es fundamental evitar las amputaciones repetidas y las zonas quirúrgicas que no cicatricen, se ha confirmado que entre el 9 y el $19 \%$ de los pacientes con una amputación por debajo de la rodilla, requieren posteriormente una amputación por encima de la misma. Por lo que respecta a la tasa de mortalidad perioperatoria, esta se encuentra entre el 5-10\% para las amputaciones realizadas por debajo de la rodilla, y entre el 10-15\% para las realizadas por encima de la rodilla (Mato, 2015). 
Es así, que los pacientes amputados de miembro inferior, usualmente restringen su participación en actividades de la vida diaria por sus limitaciones funcionales, aislándose. Igualmente, en estas personas existe un riesgo latente de exclusión social, que vulnerada su necesidad de conservar el respeto de los demás, si a esta situación se suma la falta de apoyo familiar, desempleo, entre otros, se incrementará la desvalorización de su imagen corporal, independientemente de la merma funcional sufrida.

Solo en el duelo, el dolor es absoluto, es un dolor biológico (duele el cuerpo), psicológico (duele la personalidad), social (duele la sociedad y su forma de ser), familiar (nos duele el dolor de otros) y espiritual (duele el alma). En la pérdida de un ser querido duele el pasado, el presente y especialmente el futuro (Montoya, 2007).

Este proyecto aporta en conocer los signos y síntomas existentes en cada etapa del proceso de duelo, implementando así estrategias que permita brindar un cuidado directo al paciente e indirectamente a la familia, con eficacia y eficiencia desde la enfermería, cabe indicar que es de vital importancia tener un grupo interdisciplinario donde la atención está centrada en normalizar las funciones y las acciones del paciente que han sido alteradas por el evento amputación. Para lograrlo, se necesita tener un grupo biopsicosocial, para el abordaje integral del ser humano en la que se evalúe los factores biológicos, psicológicos y sociales para la lograr el nuevo proceso de ajuste y acomodación desde su condición al medio físico, familiar, laboral y social.

Visto el problema desde esta óptica, surge como interrogante, ¿Cómo la enfermería trata al duelo pre y post quirúrgico en pacientes diabéticos que son sometidos a una amputación?, entendiendo por duelo al estado psicológico consecuente a la pérdida de un objeto significativo que formaba parte integrante de la existencia. La pérdida puede ser de un objeto externo, como la muerte de una persona, la separación geográfica, el abandono de un lugar, o interno, como la desaparición de una perspectiva, la pérdida de la propia imagen social, un fracaso personal y semejante y se concibe a la amputación como la separación o corte de un miembro o una parte del cuerpo de un ser vivo, generalmente por medio de una operación quirúrgica.

\section{Importancia del Problema}

Una amputación es un evento altamente significativo para la persona que lo sufre y para los que le rodean, el cual requieren de la atención y acompañamiento de un equipo multidisciplinario e 
interdisciplinario, en el contexto ecuatoriano se le brinda poca importancia a este evento, a través de esta investigación se ayudará a los profesionales de la salud, específicamente del área de enfermería a tomar medidas correctivas, y a plantearse nuevos programas que apoyen a mejorar la calidad de vida de los pacientes diabéticos amputados.

\section{Metodología}

La investigación es cualitativa y busca una comprensión particular del fenómeno que se estudia, entiende el significado de aquello que se muestra en lo que es diferente de los hechos, que son controlados una vez que han sido definidos, se centra en lo específico, individual y lo peculiar. La comprensión del fenómeno es lo más próximo al proceso del duelo y la vivencia del paciente, de modo que se entiende como ellos viven su experiencia, en este caso, la experiencia del paciente amputado y la percepción de su auto valencia futura. El objetivo de la investigación desde una perspectiva fenomenológica adquiere una comprensión de las estructuras esenciales de estos fenómenos sobre la base de los acontecimientos.

\section{Resultados}

El resultado de este trabajo investigativo fue enriquecedor, pues ha permitido un gran acercamiento a un grupo de poblacional con grandes necesidades de atención., mediante la investigación se pudo observar diferentes sentimientos entre ellos vergüenza y enojo mayormente, externalizados. La poca aceptación de perder una extremidad o un miembro de su cuerpo los sumerge y muchas veces los estanca en un estadio de negación llevándolos a caer en un estado depresivo, tomando en cuenta que en la actualidad los cuidados o atención de salud para estos pacientes se basan solo en tratamiento, cuidados paliativos y rehabilitación dejando de lado la Psiquis de estos pacientes.

En el gráfico 1 se muestra las causas por las cuales se produjo la amputación, demostrando que la mayoría ha llegado a estas instancias por complicaciones propias de la diabetes mellitus, siendo ésta el 75\% de las causas de amputación, seguido a ello se encuentran los accidentes de tránsito que alcanzan el 15\% y otras causas el 10\%. Visto así los resultados se demuestran claramente los efectos de la citada enfermedad, a esto se suma el deterioro de su carácter típico de los diabéticos, generando una situación difícil de superar ante la amputación de un miembro. 


\section{Gráfico 1}

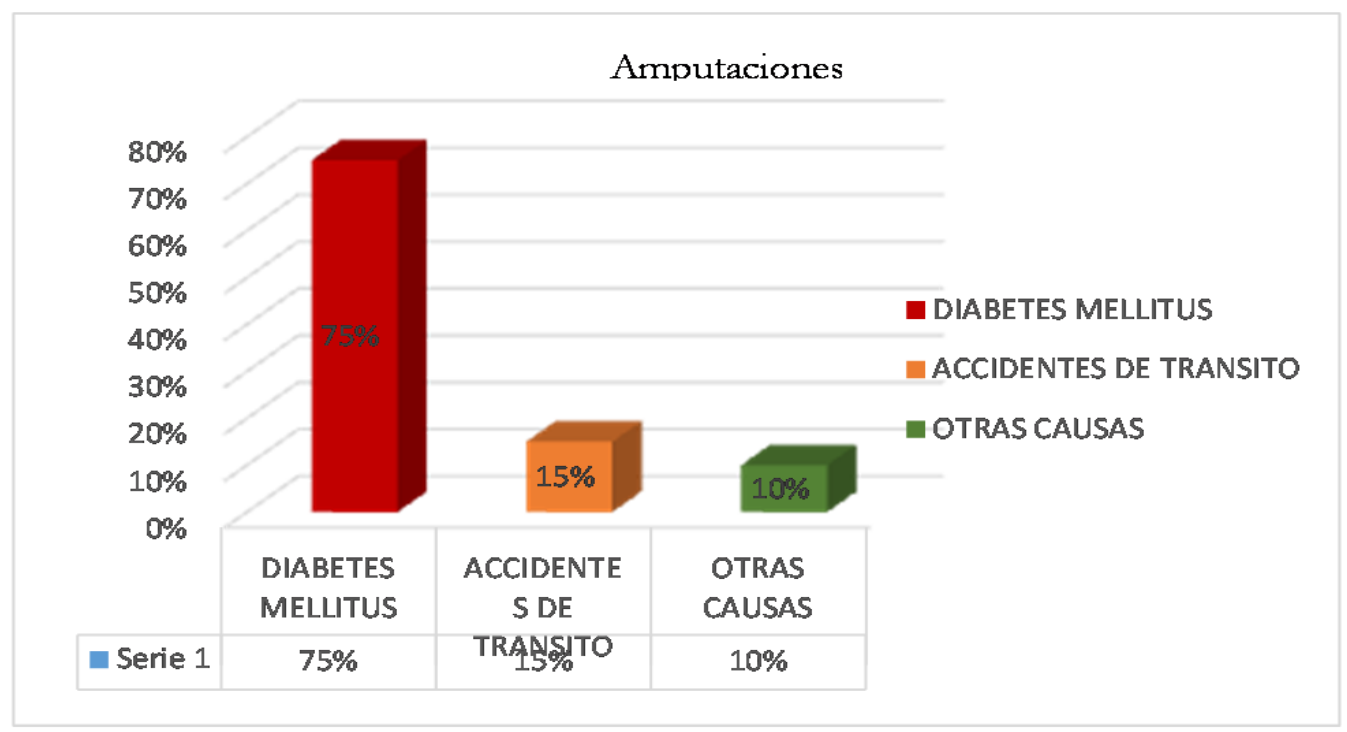

Fuente: Pacientes del Club de Diabéticos del Centro de Salud de Picoazá (2016).

En el grafico 2 se evidencia claramente el problema, dando el $60 \%$ el incumplimiento de los tratamientos, el $35 \%$ cumple con el tratamiento y el 5\% nunca, se hace indiscutible si se analizan las repercusiones que este tiene desde el punto de vista clínico, médico, económico y psicosocial.

\section{Gráfico 2}

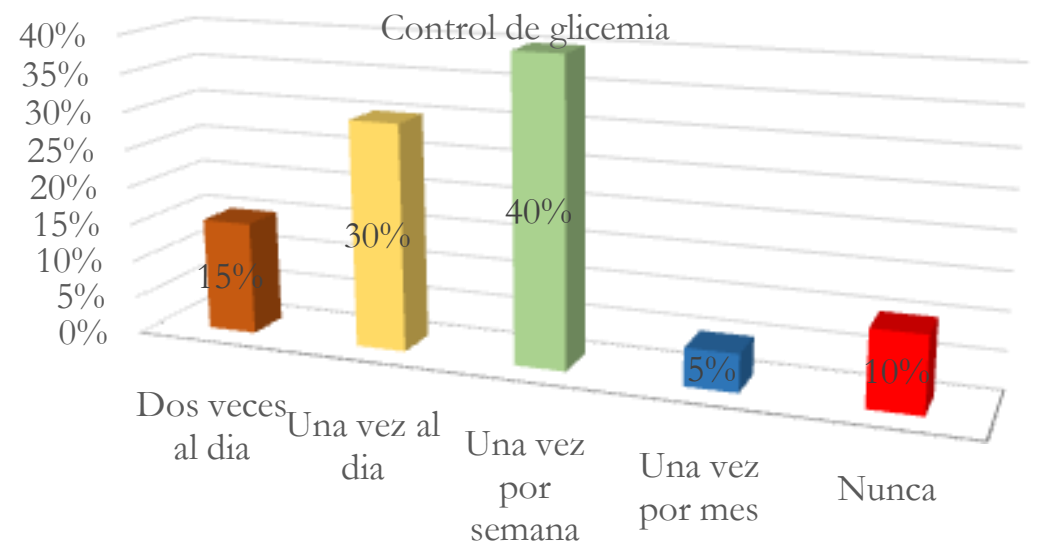

Fuente: Pacientes del Club de Diabéticos del Centro de Salud de Picoazá (2016).

En el grafico 3 es eminente y alarmante la estadística que nos da el control de la glucosa, una vez por semana con el 40\%, en comparación con el 30\% una vez al día, el $15 \%$ dos veces al día, el 
$10 \%$ nunca y el $5 \%$ una vez por mes. Los resultados nos obligan a establecer estrategias para el mejoramiento de la calidad del control en el primer nivel de atención de las áreas de salud.

\section{Gráfico 3}

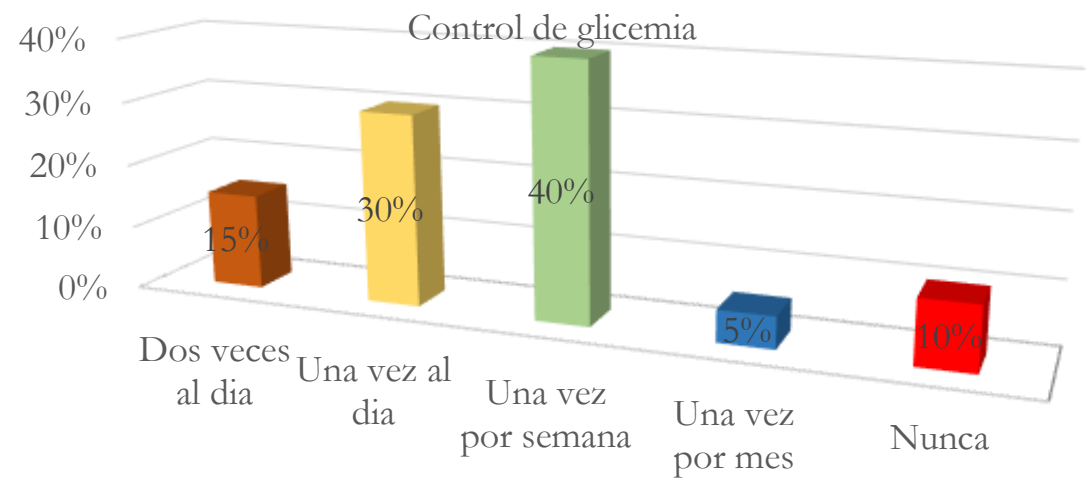

Fuente: Pacientes del Club de Diabéticos del Centro de Salud de Picoazá (2016).

En el grafico 4 demuestra que existe un $50 \%$ de pacientes que si acuden a una consulta médica ante una lesión eminente y un 50\% que no se realiza control médico.

\section{Gráfico 4}

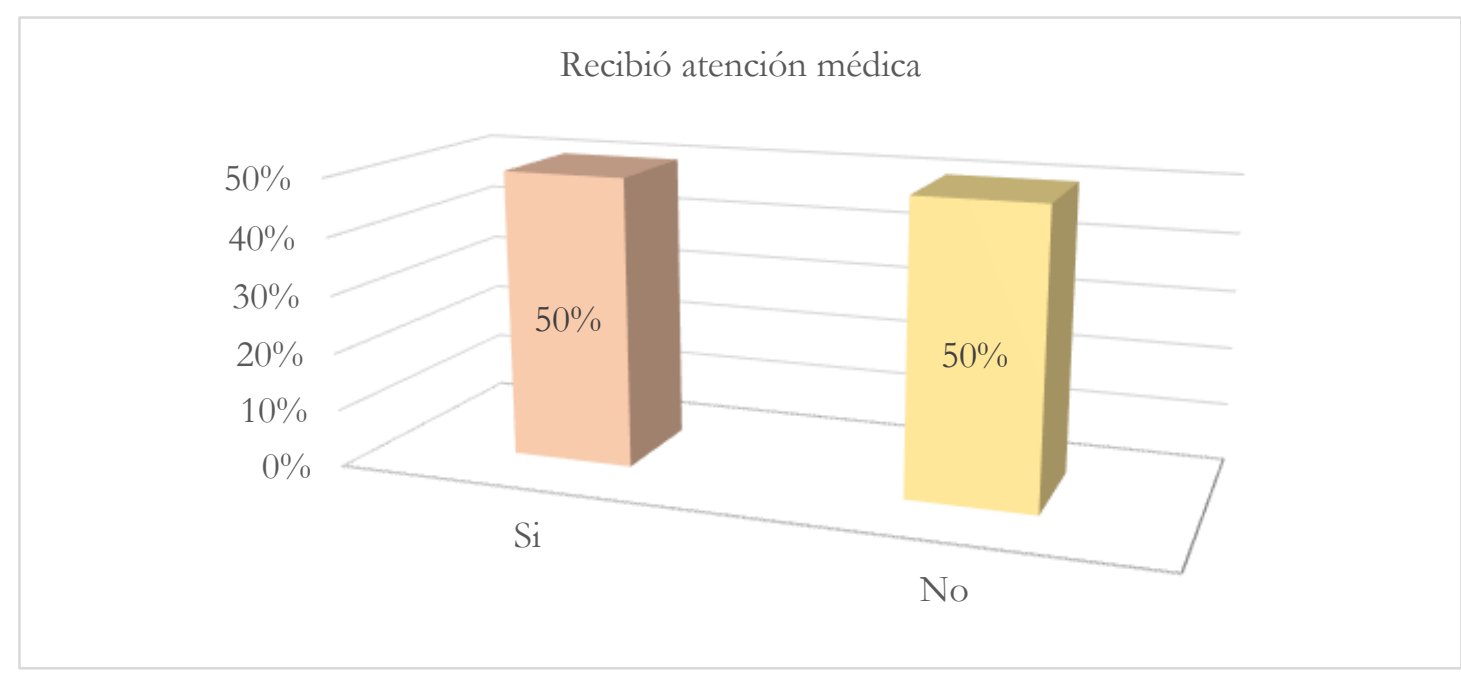

Fuente: Pacientes del Club de Diabéticos del Centro de Salud de Picoazá (2016).

\section{Discusión}

La amputación de los miembros inferiores y superiores ocurre como consecuencia del tratamiento curativo y/o paliativo ocasionado por diferentes patologías, entre las que se encuentran las causadas por alteraciones metabólicas que ocasionan problemas vasculares y traumatismos ocasionados por accidentes. Un estudio realizado en Perú demuestra el nivel de ausencia de pulso 
que refleja la gravedad de enfermedad arterial periférica y el grado de lesión (según escala de Wagner), correlacionó directamente con el riesgo de amputación.

\section{Conclusión}

La mayor causa de las amputaciones se debe a complicaciones propias de la diabetes, las consecuencias, a largo plazo, de esa enfermedad son el resultado de alteraciones de micro y macro vasculares, que pueden llevar a la disfunción de varios órganos, como: los ojos, riñones, nervios, corazón y vasos sanguíneos. Las complicaciones crónicas incluyen: retinopatía, con potencial a la perdida visual; nefropatías, con posibilidad de evolución hacia una insuficiencia renal; neuropatía periférica; con elevación progresiva en el riesgo de formación de ulceras en los pies del diabético y amputaciones.

Los pacientes que han sufrido de amputaciones poco colaboran con el tratamiento, ya que existe un riesgo eminente de exclusión social y, dependiendo de los valores esgrimidos, puede verse seriamente amenazada la necesidad que tiene toda persona de conservar el respeto de los demás y el respeto por sí mismo, cabe recalcar que la atención será brindada por un equipo de profesionales y no profesionales capacitados en este tipo de tratamiento, de acuerdo a las necesidades del paciente y la familia. En este equipo pueden participar: médicos, enfermeras, psicólogos, trabajadores sociales, acompañantes espirituales, rehabilitadores, voluntarios, familiares, amigos, educadores.

El control de la glucosa es un parámetro fundamental para el bienestar del paciente, de esta manera se controla y se detecta a tiempo las complicaciones propias de la enfermedad. Es equitativo el número de pacientes que recibieron atención médica ante la primera lesión es de vital importancia tener un grupo interdisciplinario donde la atención está centrada en normalizar las funciones y las acciones del paciente que han sido alteradas por el evento amputación. Para lograrlo, se necesita tener un grupo biopsicosocial para el abordaje integral desde las tres esferas del ser humano en la que se evalúan e intervienes los factores biológicos, psicológicos y sociales para la lograr el nuevo proceso de ajuste y acomodación desde su condición al medio físico, familiar, laboral y social.

\section{Referencias Bibliográficas}

Arevalo, B. (2010). Descripción del proceso del duelo en pacientes civiles y militares. Tesis. Universidad de Nueva Granada. Bogotá. Colombia. 
Castillo, L. (2016). Revisión Sistemática: Cuidados de Enfermería en Personas Portadoras de Dolor Fantasma de Miembro Amputado. Revista El Dolor 65. Pp. 22-28. [en línea], Disponible en: https://www.ached.cl/upfiles/revistas/documentos/580fff726c90c_original3.pdf

Díaz, A.G. (2015). Estilos de personalidad, modos de afrontamiento y clima social familiar en pacientes amputados de miembro inferior. Tesis. Universidad San Martin de Porres, Lima Perú. Mato, R. (2015). Plan de cuidados estandarizado en el postoperatorio de la amputación de miembros inferiores. tesis. Universidad Complutense de Madrid. Madrid. España.

Montoya, J. (2007). El Duelo Sobre el Cómo Ayudarnos y Ayudar a Otros a Enfrentar la Muerte de un Ser Querido. [en línea], Disponible en: http://montedeoya.homestead.com/duelos.htm.

Organización Mundial de la Salud (OMS). (2004). Departamento de Salud Mental y Abuso de Sustancias. Intervenir en salud mental. [en línea], Disponible en: http://www.who.int/mental_health/advocacy/en/spanish_final.pdf.

Organización Mundial de la Salud (OMS), (2016). Informe mundial sobre la diabetes. [en línea], Disponible en: http://www.who.int/diabetes/global-report/es/.

Instituto Nacional de Estadística y Censos (INEC), (2014). Diabetes y enfermedades hipertensivas entre las principales causas de muerte en el 2013. [en línea], Disponible en: http://www.ecuadorencifras.gob.ec/diabetes-y-enfermedades-hipertensivas-entre-las-principalescausas-de-muerte-en-el-2013/.

Organización Mundial de la Salud (OMS), (2011). Estadísticas sanitarias mundiales. [en línea], Disponible en: http://www.who.int/gho/publications/world_health_statistics/2011/es/.

Sani, Y. (2017). Propuesta de acciones de Enfermería para un cuidado paliativo al paciente diabético Lican, Riobamba. Tesis. Universidad Nacional de Chimborazo, Riobamba.

Sani, Y. (2017). Propuesta de acciones de Enfermería para un cuidado paliativo al paciente diabético Lican, Riobamba. tesis. Universidad Nacional de Chimborazo. Riobamba.

Toledo, D. (2016). Nivel de depresión en los pacientes Diabéticos e Hipertensos del Centro de Salud Biblián 2017. tesis. Cuenca: Universidad de Cuenca. 